\title{
A IMPORTÂNCIA DA COMUNICAÇÃO ENTRE A EQUIPE MULTIPROFISSIONAL PARA O PACIENTE INTERNADO NA UNIDADE DE TERAPIA INTENSIVA
}

\author{
THE IMPORTANCE OF COMMUNICATION BETWEEN THE MULTIDISCIPLINARY TEAM FOR THE \\ PATIENT ADMITTED TO THE INTENSIVE CARE UNIT
}

DOI: $10.16891 / 2317-434 X . v 10 . e 1 . a 2022 . p p 1240-1243$

Recebido em: 10.11.2021 | Aceito em: 10.11.2021

Francisco Emerson Alves da Silva, Poliana da Silva Almeida, Antônia Mayara Oliveira de Freitas, Ana Beatriz Leão Lima e Anny Karolliny Pinheiro de Sousa Luz

Centro Universitário Doutor Leão Sampaio

\section{RESUMO}

A unidade de terapia intensiva (UTI) é o ambiente hospitalar mais seguro e equipado, onde há maior controle da morte de pacientes internados. O cenário dentro da UTI oscila com muita frequência, por isso é necessária uma equipe multiprofissional, gerando interação entre as linhas de conhecimento de cada profissional, para proporcionar o melhor cuidado possível além de uma visão humanizada dos doentes. Objetivou-se aqui, aumentar as informações científicas sobre a importância da comunicação da equipe multiprofissional dentro das UTIs. Este trabalho trata-se de uma revisão de literatura realizada nas principais bases de dados e de pesquisa com os descritores: fisioterapia, integração, physiotherapy, UTI, ICU, equipe multiprofissional e multidisciplinary team nas línguas portuguesa e inglesa. Foram selecionados periódicos publicados nos últimos 8 anos (2013-2021) em idioma inglês e português. Finalmente, foram encontrados 11 artigos que se relacionam ao tema e foram utilizados no trabalho. Os estudos apontam que a comunicação entre as partes envolvidas na equipe que rege a UTI é de extrema importância para evitar erros e promover a visão humanizada dentro do setor, pois tais práticas podem contribuir para a diminuição da taxa de mortalidade e diminuição do tempo de permanência nas UTI's. No entanto, a comunicação entre a equipe pode sofrer um déficit devido a sobrecarga de trabalho e rotina agitada do setor. Dada a importância da comunicação entre a equipe multiprofissional da UTI, vê-se necessidade de mais estudos nessa temática bem como a efetivação de práticas que visem melhorar a comunicação e a integração dos profissionais da UTI.

Palavras-chave: Fisioterapia; equipe multidisciplinar; interação; integração.

\section{ABSTRACT}

The intensive care unit (ICU) is the safest and most equipped environment in hospitals, where there is greater control of inpatient deaths. The scenario within the ICU often fluctuates, which is why a multidisciplinary team is needed, generating interaction between the lines of knowledge of each professional, to provide the best possible care, in addition to a humanized view of patients. The objective here is to increase scientific information about the importance of communication by the multidisciplinary team within the ICUs. This work is a literature review carried out in the main databases and research with the descriptors: physiotherapy, integration, physiotherapy, ICU, ICU, multidisciplinary team and multidisciplinary team in Portuguese and English. Journals published in the last 8 years (2013-2021) in English and Portuguese were selected. Finally, 10 articles related to the topic were found and were used in the work. The studies show that communication between the parties involved in the team that runs the ICU is extremely important to avoid mistakes and promote a humanized view within the sector, as such practices can contribute to a decrease in the mortality rate and a reduction in the length of stay in the ICU's. However, communication between the team can suffer a deficit due to the workload and hectic routine of the sector. Given the importance of communication between the multidisciplinary team of the ICU, there is a need for more studies on this topic, as well as the implementation of practices aimed at improving communication and integration of ICU professionals.

Keyword: physiotherapy; multidisciplinary team; interaction; integration. 


\section{V.10 N.1 (2022) ISSN: 2317-434X}

\section{INTRODUÇÃO}

A unidade de terapia intensiva (UTI) é um ambiente pensado e projetado com objetivo de salvar vidas e evoluir positivamente o quadro clínico dos pacientes admitidos no setor, deixando-os o mais próximo possível de suas plenas capacidades funcionais, logo, o controle da mortalidade na UTI pode aumentar e prolongar a vida dos pacientes nela admitidos (DE SOUZA SOARES et al., 2019).

São comuns na UTI, quadros emergenciais, que demandam raciocínio clínico de forma rápida, além de ações decisivas por parte da equipe que rege o ambiente. Por esse motivo, os constituintes da equipe multiprofissional precisam alinhar seus conhecimentos em prol do benefício do paciente em situação emergencial, bem como pacientes críticos. Para se obter resultados positivos nas situações supracitadas há a necessidade da atualização científica constante por parte dos profissionais, a fim de desenvolver habilidades, uma boa interação profissional e aprendizados que passam a ser necessários e aplicados na prática clínica (SILVA et al., 2019).

A UTI requer em sua equipe multiprofissional pelo menos um médico para cada dez leitos, mais dois médicos sendo um rotineiro e um plantonista, pelo menos um enfermeiro plantonista para cada dez leitos, e mais dois sendo um coordenador e outro rotineiro, um fisioterapeuta plantonista, um fisioterapeuta responsável técnico, um fonoaudiólogo, um psicólogo, um técnico de enfermagem para cada dois leitos por turno, pelo menos um auxiliar administrativo exclusivo da unidade, e funcionários exclusivos para desempenharem o serviço de limpeza (DE SOUZA SOARES et al., 2019).

A presente pesquisa tem por objetivo aumentar e propagar informações científicas acerca da importância da comunicação entre os profissionais que constituem a equipe multiprofissional dentro das unidades de terapia intensiva para os pacientes admitidos no setor.

\section{METODOLOGIA}

O presente trabalho trata-se de uma revisão integrativa da literatura sobre a importância da comunicação entre os profissionais que formam equipe multiprofissional atuante na unidade de terapia intensiva e debate sobre a importância dessa comunicação mais efetiva para os pacientes admitidos nesse setor.

Foram incluídos no estudo artigos publicados no período de 2013 a 2021, em idiomas inglês e português, que compuseram os resultados das buscas feitas pelos seguintes descritores: fisioterapia, integração, physiotherapy, UTI, ICU, equipe multiprofissional e multidisciplinary team nas principais bases de dados: PEDro, google acadêmico, PubMed e Scielo. Ao fim da pesquisa foram encontrados 11 artigos que se relacionam ao tema e foram utilizados no trabalho.

\section{RESULTADOS E DISCUSSÕES}

Instaurada no ano de 2003, a Política Nacional de Humanização (PNH) explicita a importância do acolhimento humanizado ao paciente e seu familiar, além de aclarar a importância da comunicação e relações interpessoais da equipe multiprofissional (GOULARTE et al., 2020).

A UTI é o campo de atendimento médico que realiza atendimentos de alta complexidade, lidando com os mais delicados e críticos casos, através de técnicas avançadas de suporte de vida (DE SOUZA, 2020). Deste modo, é indispensável que a equipe multidisciplinar utilize da comunicação ativa como estratégia da construção do vínculo e confiança, proporcionando melhor qualidade de vida ao paciente em cuidados intensivos (SILVA, 2019).

$\mathrm{O}$ ambiente da UTI acaba sendo considerado estressante pelos pacientes, familiares e até equipe multiprofissional responsável. Há a probabilidade que essa classificação feita pelas pessoas que ocupam a unidade de terapia intensiva se deva às oscilações, muitas vezes constantes, dos cenários e contextos clínicos por se tratar de pacientes críticos (SILVA et al., 2021). Com a falta de comunicação interprofissional, abertura para comunicações deficientes, escassez de interação pacienteequipe e fragilidades na comunicação durante passagens de plantões e transferência do paciente, torna-se um dos principais pontos de risco ao paciente, podendo levar a uma experiência ruim no processo de cuidado, afetando a sua segurança e os seus resultados clínicos (SANTOS et al., 2021).

Os colaboradores da UTI sofrem diariamente desgastes físicos e psicológicos devido às longas jornadas de trabalho e as situações de altas complexidade que muitas vezes envolve dor, sofrimento, mal-estar físico e emocional dos pacientes (SANTANA et al., 2016; PÊGO et al., 2016) levando a equipe a conflitos comportamentais como má comunicação e até abusos verbais (MOON e KIM, 2015). Os profissionais que atuam em UTI convivem diariamente com a dificuldade da aceitação da morte, a escassez de recursos materiais e de recursos humanos e a tomada de decisões conflitantes relacionadas com a seleção dos pacientes que serão atendidos, fatores estes que geram desgastes. Estas são algumas das situações em que ocorre tensão entre os profissionais e acaba por influenciar de forma negativa, a qualidade da 
assistência prestada aos pacientes (DE SOUZA SOARES et al., 2019).

Um estudo realizado por Amaral e Oliveira (2016), descrevia uma intervenção grupal realizada com dez profissionais de uma equipe de saúde de uma Unidade de Terapia Intensiva Coronariana, e com a finalização da intervenção foi possível observar maior facilidade na comunicação entre todos os profissionais, além de valorização do autocuidado por parte da equipe.

Com objetivo de analisar o conhecimento da equipe multiprofissional acerca da importância da mobilização precoce em pacientes adultos graves, Fontela Junior e Friendman (2018), após investigar uma amostra de 98 profissionais, puderam perceber o reconhecimento da importância da mobilização precoce bem como seus benefícios, entretanto também foram identificadas barreiras relacionadas a rotina de trabalho, interação da equipe, funcionamento da UTI e situação clínica dos pacientes.

\section{CONCLUSÃO}

Visto todos os fatores que fazem da comunicação um ponto de grande importância a ser desenvolvido e discutido entre os profissionais que compõem a equipe multiprofissional da UTI, denota-se a necessidade de desenvolvimento de mais estudos voltados para essa temática, bem como a implementação efetiva dentro do cenário da UTI de práticas que visem o melhoramento da comunicação e a integração da equipe multiprofissional da unidade de terapia intensiva.

\section{REFERÊNCIAS}

AMARAL, SRC; OLIVEIRA, AEG. Grupo de reflexão com profissionais de uma Unidade de Terapia Intensiva Coronariana: um relato de experiência. Rev. bras. saúde ocup. 2016; $21(\mathrm{e} 24): 1-8$. http://www.scielo.br/pdf/rbso/v41/2317-6369-rbso-41e24.pdf

DE SOUZA SOARES, Anne Louise; CONCEIÇÃO, Tamires Pantoja; MONTEIRO, Valéria Renata. A INTEGRAÇÃO DA EQUIPE MULTIPROFISSIONAL NA UNIDADE DE TERAPIA INTENSIVA: REVISÃO SISTEMÁTICA. Revista CPAQV-Centro de Pesquisas Avançadas em Qualidade de Vida| Vol, v. 11, n. 3, p. 2, 2019.

DE SOUZA, Tácyla Medeiros et al. Papel da comunicação em saúde frente aos cuidados paliativos na unidade de terapia intensiva. Brazilian Journal of Development, $v$. 6, n. 11, p. 93059-93066, 2020.

FONTELA, PC; FORGIARINI JUNIOR, LA; FRIEDMAN G. Atitudes clínicas e barreiras percebidas para a mobilização precoce de pacientes graves em unidades de terapia intensiva adulto. Rev. bras. ter. intensiva. 2018. Jun. 30(2):187-94. http://www.scielo.br/pdf/ rbti/v30n2/0103-507X-rbti-3002-0187.pdf

GOUlaRTE, P. N., Gabarra, L. M., \& Moré, C. L.
O. (2020). A visita em Unidade de Terapia Intensiva adulto: perspectiva da equipe multiprofissional. Rev. Psicol. Saúde [online]. 12(1), 157 170. DOI: 10.20435 / pssa.v12i1.734.

MOON JY, KIM J-O. Ethics in the Intensive Care Unit. Tuberc Respir Dis. 2015;78(3):175-9. doi: 10.4046/trd.2015.78.3.175

PÊGO, FPL; PÊGO DR. Síndrome de Burnout. Rev Bras Med Trab. 2016;14(2):171-6. DOI:10.5327/Z1679443520162215.

SANTANA, LL; SARQUIS, LMM; MIRANDA, FMD; KALINKE, LP; FELLI, VEA; MININEL, VA; et al. Health indicators of workers of the hospital area. Rev Bras Enferm [Internet]. 2016 DOI: 10.1590 / 00347167.2016690104i

SANTOS, José Augustinho Mendes et al. Comunicação e segurança do paciente em Unidade de Terapia Intensiva: perspectivas da equipe multiprofissional de saúde. Research, Society and Development, v. 10, n. 13, p. e131101320898-e131101320898, 2021.

SILVA, Andressa Fernanda et al. Presenteeism in multiprofessional team workers in the Adult Intensive Care Unit. Revista Brasileira de Enfermagem [online]. 2019, v. 72, suppl 1 [Acessado 8 Novembro 2021], pp. 96104. Disponível em: <https://doi.org/10.1590/0034-7167- 


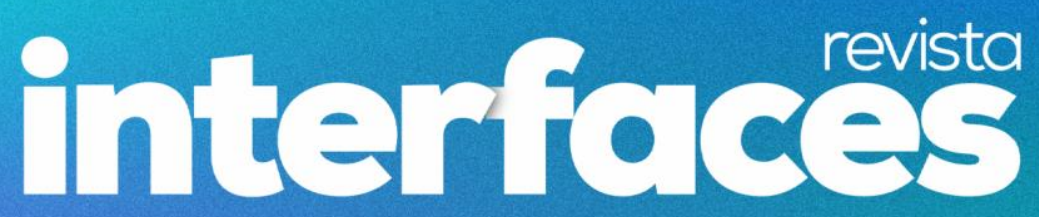

Resumos

V.10 N.1 (2022) ISSN: 2317-434X

2017-0779>.

ISSN

1984-0446.

https://doi.org/10.1590/0034-7167-2017-0779.

SILVA, KV.; GOMES, AM de A..; MAIA, MA de Q. Saberes e práticas do cuidado humanizado por equipe multiprofissional em uma Unidade de Terapia Intensiva Coronariana - UTI. Pesquisa, Sociedade e Desenvolvimento, [S. 1.] , v. 10, n. 8, pág. e42210817390, 2021. DOI: 10.33448 / rsd-v10i8.17390. Disponível em: https://rsdjournal.org/index.php/rsd/article/view/17390.

Acesso em: 8 nov. 2021. 